Fabrizio Vianello, Luisa Sambado, Pamela Scarparo, Annamaria Lombardi, Daniela Bernardi, Mario Plebani and Fabrizio Fabris*

\title{
Comparison of three different immunoassays in the diagnosis of heparin-induced thrombocytopenia
}

DOI 10.1515/cclm-2014-0809

Received August 25, 2014; accepted September 27, 2014; previously published online October 17, 2014

\section{Abstract}

Background: Heparin-induced thrombocytopenia (HIT) is caused by platelet activating antibodies that recognize platelet factor 4/heparin $(\mathrm{PF} 4 / \mathrm{H})$ complexes. Laboratory testing plays a key role in the diagnosis of HIT. As functional assays are unfeasible for most clinical laboratories, antigen binding assays are commonly used in routine testing. However, their low specificity leads to overdiagnosis of HIT. Therefore, it is advisable to improve screening tests in this setting.

Methods: Blood samples from 114 patients in whom HIT was suspected were investigated using a chemiluminescence test (HemosIL ${ }^{\circledR}$ AcuStar HIT-IgG), a PF4/H IgG enzyme immunoassay (Lifecodes PF4 IgG), an IgG-specific lateral flow immunoassay heparin-induced thrombocytopenia (LFI-HIT, STic Expert ${ }^{\circledR}$ HIT) and the heparin-induced platelet aggregation (HIPA) test.

Results: Twenty-nine (25.4\%) out of 114 subjects with suspected HIT had a positive HIPA test. None of patients with a 4Ts score $<4$ were positive at HIPA. HemosIL ${ }^{\circledR}$ AcuStar HIT-IgG showed the best performance in term of sensitivity and specificity when used as single test. Receiver operating characteristic (ROC) analysis showed optimization of sensitivity and specificity using a cut-off of $1.13 \mathrm{U} / \mathrm{mL}$ (0.95 and 0.98, respectively). As an alternative approach, a strategy based on screening samples by STic Expert ${ }^{\circledR}$ HIT and then retesting positive results by Lifecodes PF4 IgG

*Corresponding author: Fabrizio Fabris, MD, Department of Medicine, Internal Medicine Unit University-Hospital of Padua, Via Giustiniani 2, 35128 Padua, Italy, Phone: +39 0498212150, Fax: +39 0498217823, E-mail: fabrizio.fabris@unipd.it Fabrizio Vianello, Luisa Sambado, Pamela Scarparo and Annamaria Lombardi: Department of Medicine, Internal Medicine Unit, University-Hospital of Padua, Padua, Italy

Daniela Bernardi and Mario Plebani: Department of Laboratory Medicine, University-Hospital of Padua, Padua, Italy (cut-off 1 OD) or HemosIL ${ }^{\circledR}$ AcuStar HIT-IgG (cut-off 1.3 $\mathrm{U} / \mathrm{mL}$ ) showed a performance compared to a single test approach by HemosIL ${ }^{\circledR}$ AcuStar HIT-IgG.

Conclusions: The HemosIL ${ }^{\circledR}$ AcuStar HIT or a combinatorial approach with the STic Expert ${ }^{\circledR}$ HIT and the PF4/H IgG enzyme immunoassay provide an accurate diagnosis of immune HIT.

Keywords: clinical laboratory techniques; heparin; platelet factor 4; thrombocytopenia.

\section{Introduction}

Heparin is widely used for the prevention and treatment of thromboembolic disorders. Unfortunately, during heparin therapy patients may develop heparin-induced thrombocytopenia (HIT), a prothrombotic disorder mediated by IgG antibodies recognizing heparin-bound platelet factor 4 (PF4). The generation of these macrocomplexes triggers thrombin generation responsible for life threatening venous and arterial thromboembolic complications [1, 2].

Although suspicion of HIT is based on a well-defined clinical scenario, in vitro demonstration of heparin dependent pathogenic antibodies confirms or refutes the diagnosis.

Functional and antigen assays are available for the laboratory diagnosis of HIT and the use of both tests seem to increase the accuracy in confirming the clinical diagnosis [3]. Functional assays include several methods able to show the capacity of patient sample to activate selected donor's platelets in presence of therapeutic doses of heparin. In this field the gold standard is represented by the serotonin release assay (SRA) or the heparin-induced platelet activation assay (HIPA). As these tests are technically challenging and not widely available, enzyme immunoassays (EIAs) have had an increasingly diagnostic application especially in Europe [3]. Enzyme immunoassay is sensitive, easier and faster than functional tests; however, this method has a lower specificity [4]. 
The primary aim of the present work was to compare the performance of three commercially available immunoassays for anti-PF4/heparin $(\mathrm{H})$ antibodies in predicting the presence of HIT antibodies activating platelets in patients with suspected HIT. Sequential testing was also evaluated with the aim to maximize the diagnostic specificity without at the same time compromising sensitivity.

\section{Materials and methods}

We evaluated 114 consecutive patients with a clinical diagnosis of HIT according to the 4Ts score [5]. Blood samples referred to our centre were centrifuge and plasma and serum aliquots were stored at $-80^{\circ} \mathrm{C}$. All 114 samples were tested using an EIA for PF4/H IgG (Lifecodes PF4 IgG, Immucor, Milan, Italy), by a rapid lateral flow immunoassay (LFIA) (H/PF4 STic Expert ${ }^{\circledR}$ HIT, Diagnostica Stago SAS, Asnieres sur Seine, France; $n=114)$, whereas fewer samples (96/114) were tested by the chemiluminescence assay HemosIL ${ }^{\circledR}$ AcuStar HITIgG (PF4/H) (Instrumentation Laboratory, Bedford, MA, USA). As reference test in the study, the HIPA test was performed in all samples.

HIPA test was performed according to Greinacher et al. [6]. Briefly, platelet-rich plasma (PRP), obtained from normal donor was supplemented with $111 \mu \mathrm{L} / \mathrm{mL}$ of ACD and $5 \mu \mathrm{L} / \mathrm{mL}$ of Apyrase $1000 \mathrm{U} / \mathrm{mL}$ (Sigma, Munich). After centrifugation for $7 \mathrm{~min}$ at $650 \mathrm{~g}$, platelets were resuspended in $5 \mathrm{~mL}$ of washing buffer $(10 \mathrm{~mL}$ tyrode buffer $25 \mathrm{U}$ apyrase $10 \mathrm{U}$ hirudin $\mathrm{pH} 6.3$ ), incubated for $15 \mathrm{~min}$ at $37^{\circ} \mathrm{C}$ and then centrifuged. The pellet was resuspended in $1 \mathrm{~mL}$ of resuspension buffer $\left(50 \mathrm{~mL}\right.$ of tyrode buffer, $0.212 \mathrm{M} \mathrm{MgCl}_{2}$, and $0.196 \mathrm{M}$ $\mathrm{CaCl}_{2}, \mathrm{pH}$ 7.2). Donor platelets were adjusted to 300,000 platelets $/ \mu \mathrm{L}$. Patients' serum was heat inactivated $\left(30 \mathrm{~min}\right.$ at $\left.56^{\circ} \mathrm{C}\right)$ and $20 \mu \mathrm{L}$ were added to a microtiter plate, supplemented with $10 \mu \mathrm{L}$ of $0.2 \mathrm{U} / \mathrm{mL}$ or $100 \mathrm{U} / \mathrm{mL}$ of UFH, and $75 \mu \mathrm{L}$ of platelets from donors. During the incubation wells were controlled for loss of turbidity at 5-min intervals. A sample was considered positive for HIT antibodies when the well turned transparent with $0.2 \mathrm{U} / \mathrm{mL}$ UFH but not with heparin $100 \mathrm{U} / \mathrm{mL}$, using platelets from at least two donors.

The Lifecodes PF4 IgG detects serum antibodies bound to immobilized target antigen, a complex between the polyanionic substrate polyvinyl sulfonate (PVS) and PF4. The PVS surface serves in the solid phase as a surrogate for heparin. Serum samples from patients and the control were prepared as indicated by the supplier. Samples were considered as weakly positive or positive for OD values 0.400-0.999 or $\geq 1$, as previously suggested [4]. The intra-assay precision (precision within an assay) was calculated from three pathological samples and three normal samples in a single assay run. The inter-assay precision (precision between assays) was calculated in the same set of samples in five separate runs, carried out on different days. Results expressed as coefficient of variance for this test were $2.7 \pm 0.63$ and $3.2 \pm 0.9$, respectively.

The HemosIL ${ }^{\circledR}$ AcuStar HIT-IgG (PF4/H) is a chemiluminescent two-step immunoassay detecting the presence of anti-PF4/H antibodies [7]. Briefly, plasma was incubated with latex beads coated with PF4/polyvinilsulfonate complex. After incubation, and magnetic separation a tracer (isoluminol labeled antihuman IgG antibody) was added to the samples. Following magnetic separation, chemioluminescent reagent was added and measured with the appropriate light unit by optical system ACL Acustar. The signal is directly proportional to the anti- $\mathrm{PF} 4 / \mathrm{H}$ antibody concentration in the sample. A result $>1 \mathrm{U} / \mathrm{mL}$ was considered as positive. For this test, coefficients of variance were $<2 \%$.

The H/PF4 STic Expert ${ }^{\circledR}$ HIT uses a reagent buffer composed of $\mathrm{PF} 4 /$ polyanion complexes linked to biotin and a membrane containing gold nanoparticles coated with anti-biotin antibodies as well as goat anti human IgG immobilized on the test line [8]. Briefly, $5 \mu \mathrm{L}$ of serum were added with two drops of reagent on the test strip. During the migration through the membrane, the PF4/polyanion complex binds the immobilized conjugate, gold nanoparticles coated with a polyclonal goat antibody against Fc part of human IgG, to form the detection line. After $10 \mathrm{~min}$ the test line was compared with an evaluation card. Every strip is provided with an internal control. A result was evaluated as negative when the test line (Line-T) was not visible, or was lighter coloured or weaker than the reference line on the Evaluation Card. The result was evaluated as positive when the Line-T was similar to, or darker than, the reference line. The interreader reproducibility (by two different technicians) evaluated by $\kappa$ ratio was 0.95 .

The Local Institutional Review Board approved the protocol. The study was conducted in accordance with the Declaration of Helsinki.

\section{Statistical analysis}

HIT was diagnosed in patients with an intermediate or high probability 4Ts score $(\geq 4)$ and a positive HIPA. The performance of the three assays was evaluated with respect to the reference standard by ROC analysis. Areas under the ROC curves (AUCs) were calculated and compared by the De Long method for correlated samples [9].

Analyses were carried out using GraphPad Prism 5 (GraphPad Software, La Jolla, CA, USA). A p-value $\leq 0.05$ was considered statistically significant.

\section{Results}

The clinical characteristics of the 114 patients studied are shown in Table 1.

In our cohort of 114 patients evaluated for suspected HIT, 29 (25.4\%) had a positive HIPA test. Each of these 29 patients had a 4Ts score $\geq 4$ and therefore they have been considered as HIT-positive patients. All other patients tested negative for HIPA and were considered negative for HIT. Comparison between HIT-negative and -positive patients showed a significantly greater 4Ts score in HITpositive cases $(\mathrm{p}<0.0001$, Table 1$)$ and a significantly higher rate of thrombosis compared to the HIT-negative group $(p<0.0001$, Table 1$)$. None of patients with a 4Ts score $<4$ were positive at HIPA whereas 38 patients with a 4 Ts score $>4$ were HIPA-negative.

All 114 patients were tested using the Lifecodes PF4 IgG and STic Expert ${ }^{\circledR}$ HIT immunoassays whereas in 18 out of 114 subjects we were not able to perform the 
Table 1 Demographic and clinical characteristics of HIT-positive and -negative subjects.

\begin{tabular}{lrrr}
\hline Characteristic & $\begin{array}{rrr}\text { HIT positive } \\
\mathbf{n = 2 9}\end{array}$ & $\begin{array}{r}\text { HIT negative } \\
\mathbf{n = 8 5}\end{array}$ & p-Value \\
\hline Age, mean (range) & $70(36-90)$ & $62.2(20-97)$ & 0.07 \\
Female, $\mathrm{n}(\%)$ & $12(41.4)$ & $48(56.5)$ & 0.16 \\
Medicine $\mathrm{n}(\%)$ & $7(24)$ & $38(44.7)$ & 0.06 \\
Orthopedic & $4(13.8)$ & $4(4.7)$ & 0.08 \\
Cardiovascular & $10(34.5)$ & $13(15.3)$ & 0.05 \\
surgery & & & \\
Intensive care & $6(20.7)$ & $13(15.3)$ & 0.45 \\
Others & $1(3.5)$ & $7(8.2)$ & 0.41 \\
Platelet count $\times 10^{\%} / \mathrm{L}$, & $48(32-85)$ & $57(31-97)$ & 0.19 \\
median (range) & & & \\
4Ts score, median & $6(4-8)$ & $3(1-6)$ & $<0.0001$ \\
(interquartile range) & & & \\
4Ts score $\geq 4, \mathrm{n}(\%)$ & $29(100)$ & $36(42.3)$ & $<0.0001$ \\
Thrombosis, $\mathrm{n}(\%)$ & $15(51.7)$ & $2(2.3)$ & $<0.0001$ \\
\hline
\end{tabular}

HemosIL ${ }^{\circledast}$ AcuStar HIT-IgG assay due to lack of plasma samples. Figure 1 shows the results of the Lifecodes PF 4 IgG and HemosIL ${ }^{\circledR}$ AcuStar HIT-IgG for HIT-negative and -positive cases. Both immunoassays showed significantly lower mean concentrations in HIT-negative compared to HIT-positive samples (Lifecodes PF4 IgG 0.4618 vs. 2.930,

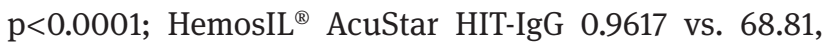
$\mathrm{p}<0.0001)$.

Table 2 shows the sensitivity/specificity pairs for each immunoassay at the manufacturer recommended cut-off. For the Lifecodes PF4 IgG, the cut-off $>1.00$ was also evaluated as it has been previously shown to increase specificity [4].

At the recommended cut-off of $0.4 \mathrm{OD}$, the sensitivity and specificity of Lifecodes PF4 IgG was 1 and 0.59, respectively, and it correctly classified 69\% (79/114) of samples. The same assay at a cut-off of 1 OD showed a sensitivity of 0.89 with a specificity of 0.88 , with $88.5 \%$ of patients correctly diagnosed, therefore with $11.5 \%$ of false negative cases.

The sensitivity and specificity of HemosIL ${ }^{\circledR}$ AcuStar HIT-IgG at cut-off of $1 \mathrm{U} / \mathrm{mL}$ were 0.95 and 0.93 , respectively, and were associated with correct classification of $93.7 \%$ (90/96) of samples, therefore with $6.3 \%$ of cases misdiagnosed as negative.

One hundred and three out of 114 (90.4\%) samples were correctly diagnosed with STic Expert ${ }^{\circledR}$ HIT, with a sensitivity and specificity of 0.93 and 0.89 , respectively (Table 2). With this assay, the rate of false negative cases was $9.6 \%$.

We next analyzed the predictive values of HemosIL ${ }^{\circledR}$ AcuStar HIT-IgG and Lifecodes PF4 IgG using receiver
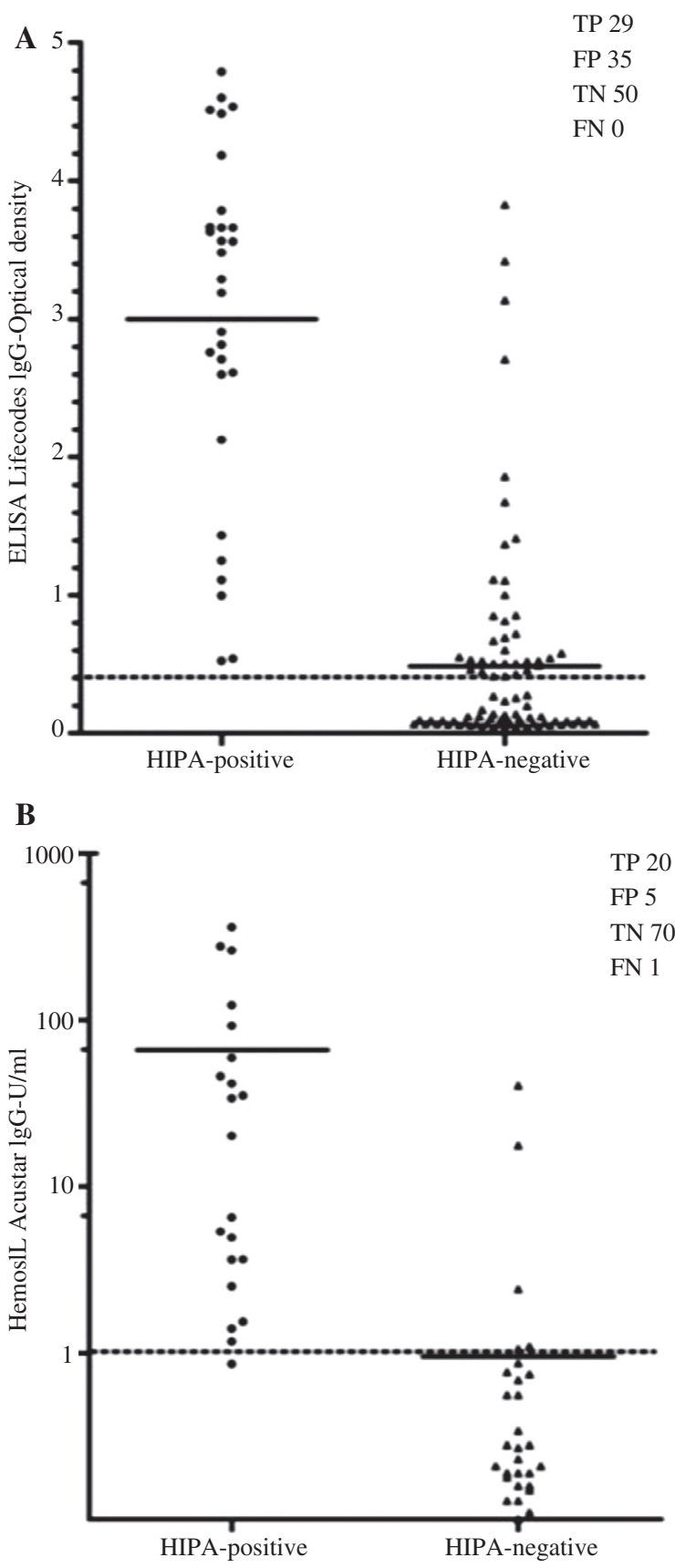

Figure 1 Assay results in HIT-negative and -positive subjects. Results from HIT-negative and -positive subjects are shown for the Lifecodes PF4 IgG (A) and HemosIL ${ }^{\circledR}$ AcuStar HIT-IgG (B). Solid horizontal lines represent mean values. Dotted lines refer to cut-offs. FN, false negatives; FP, false positives; TP, true positives; TN, true negatives.

operating characteristics (ROC) (Figure 2). The area under the curve (AUC) for the HemosIL ${ }^{\circledR}$ AcuStar HIT-IgG was greater than the AUC for the Lifecodes PF4 IgG test $(0.981 \%$ 95\% CI 0.98-1.003; 0.956\%, 0.93-0.99, respectively).

The best compromise between specificity and sensitivity was evaluated by extrapolating the most northwest 
Table 2 Results of the three immunoassays in patients with and without heparin-induced. Thrombocytopenia (4Ts Score $\geq 4$ and HIPA positive test).

\begin{tabular}{|c|c|c|c|c|c|c|c|c|}
\hline \multirow[t]{2}{*}{ Test } & \multicolumn{2}{|c|}{ Positive test } & \multicolumn{2}{|c|}{ Negative test } & \multirow[t]{2}{*}{ Specificity } & \multirow[t]{2}{*}{ Sensitivity } & \multirow[t]{2}{*}{ NPV } & \multirow[t]{2}{*}{ PPV } \\
\hline & True & False & True & False & & & & \\
\hline $\begin{array}{l}\text { Lifecodes PF4 IgG OD>0.4 } \\
(\mathrm{n}=114)\end{array}$ & 29 & 35 & 50 & 0 & 0.59 & 1 & 1 & 0.45 \\
\hline $\begin{array}{l}\text { Lifecodes PF4 IgG OD>1 } \\
(n=114)\end{array}$ & 25 & 10 & 76 & 3 & 0.88 & 0.89 & 0.96 & 0.71 \\
\hline $\begin{array}{l}\text { HemosIL }{ }^{\circledR} \text { AcuStar HIT-IgG } \\
(n=96)\end{array}$ & 20 & 5 & 70 & 1 & 0.93 & 0.95 & 0.99 & 0.8 \\
\hline $\begin{array}{l}\text { STic Expert }{ }^{\circledR} \text { HIT } \\
(n=114)\end{array}$ & 27 & 9 & 76 & 2 & 0.89 & 0.93 & 0.97 & 0.75 \\
\hline
\end{tabular}
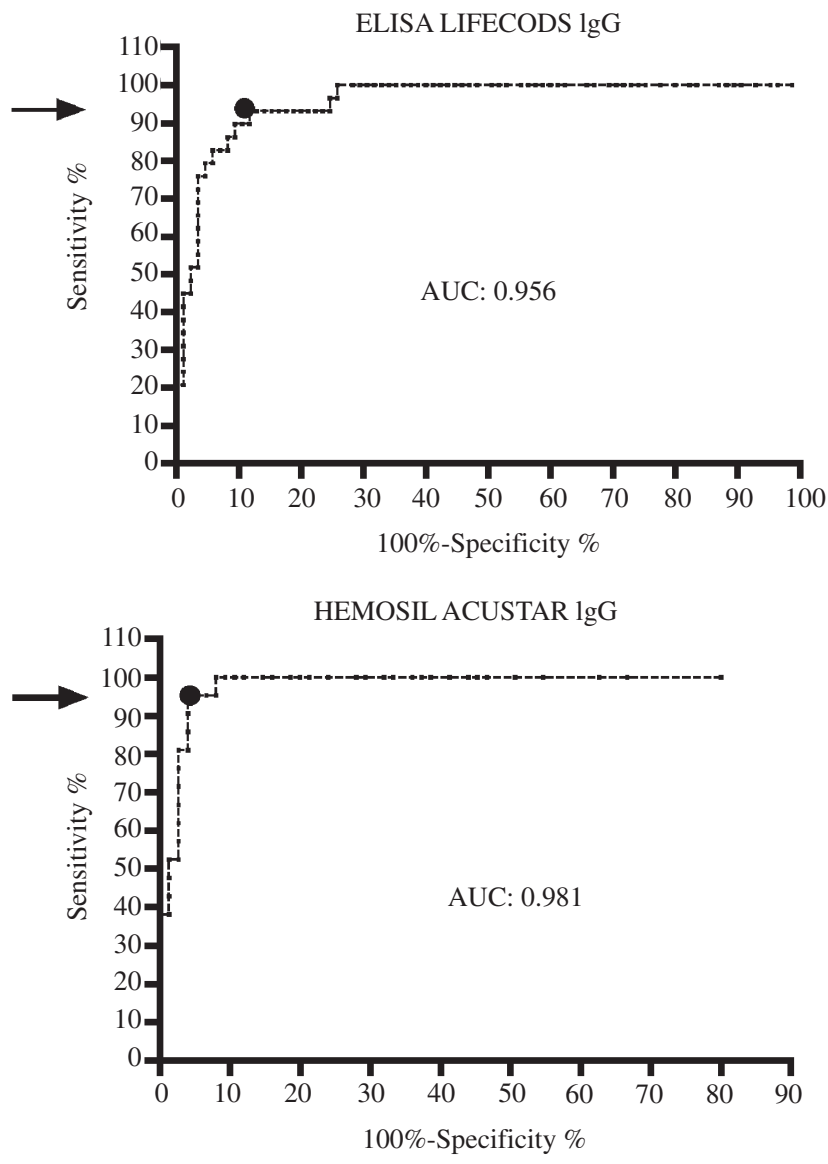

Figure 2 ROC curves.

ROC curves are shown for the Lifecodes PF4 IgG and for the HemosIL ${ }^{\circledR}$ AcuStar HIT-IgG. The AUCs for these assays are shown. Arrow: suggested cut-off.

point of the ROC curves. At a cut-off of $0.92 \mathrm{OD}$, the sensitivity and specificity of Lifecodes PF4 IgG was 0.93 and 0.88 , respectively, with a remarkably increase of specificity and a slight impairment of the sensitivity compared to the cut-off of $0.4 \mathrm{U} / \mathrm{mL}$. The best combination of sensitivity and specificity with a single test was observed with HemosIL ${ }^{\circledR}$ AcuStar HIT-IgG at the suggested cut-off of $1 \mathrm{U} / \mathrm{mL}$ (Table 2). With this assay, ROC analysis found an optimization of sensitivity and specificity using a cut-off $1.13 \mathrm{U} / \mathrm{mL}$ (0.95 and 0.96, respectively).

We then assessed whether combinatorial sequential testing could improve the specificity of each immunoassays considered for the study. Samples, which tested positive after the first screening, were tested with a second assay. Results are summarized in Table 3. None of the sequential testing performed better that HemosIL ${ }^{\circledR}$ AcuStar HIT-IgG as single test at a cut-off of $1.13 \mathrm{U} / \mathrm{mL}$. A strategy based on screening samples by STic Expert ${ }^{\circledR}$ HIT and then retesting positive results by Lifecodes PF4 IgG (cut-off 1 OD) or HemosIL ${ }^{\circledR}$ AcuStar HIT-IgG (cut-off 1.3 $\mathrm{U} / \mathrm{mL}$ ) showed a better performance compared to a single test approach by STic Expert ${ }^{\circledR}$ HIT or Lifecodes PF4 IgG (Table 3). However, this sequential approach did not significantly differ when compared to HemosIL ${ }^{\circledR}$ AcuStar HIT-IgG used as single test.

\section{Discussion}

The diagnosis of HIT is based on clinical features and on in vitro demonstration of heparin dependent antibodies as revealed by immune or functional laboratory assays. The main concern in the determination of anti-PF4/H antibodies is the overdiagnosis of HIT as a consequence of low specificity of available immunoassays. In fact, only a fraction of patients with $\mathrm{PF} 4 / \mathrm{H}$ antibodies detected by immunoassays will develop the clinical syndrome [10, 11]. Therefore, optimization of diagnostic specificity is a relevant goal of the research in the field. This can be accomplished by identifying a set of cut-offs associated with the highest probabilities of detecting platelet activating and 
Table 3 Sensitivity and specificity of sequential immunoassay approach.

\begin{tabular}{|c|c|c|c|c|c|c|c|c|}
\hline \multirow[t]{2}{*}{ Test } & \multicolumn{2}{|c|}{ Positive test } & \multicolumn{2}{|c|}{ Negative test } & \multirow[t]{2}{*}{ Specificity } & \multirow[t]{2}{*}{ Sensitivity } & \multirow[t]{2}{*}{ NPV } & \multirow[t]{2}{*}{ PPI } \\
\hline & True & False & True & False & & & & \\
\hline $\begin{array}{l}\text { STic Expert }{ }^{\circledast} \text { HIT + AND } \\
\text { Lifecodes PF4 IgG S >1 OD }\end{array}$ & 27 & 7 & 78 & 2 & 0.918 & 0.931 & 0.975 & 0.794 \\
\hline $\begin{array}{l}\text { STic Expert }{ }^{\circledR} \text { HIT + AND } \\
\text { HemosIL }{ }^{\circledR} \text { AcuStar HIT-IgG }>1.3 \mathrm{U} / \mathrm{mL}\end{array}$ & 19 & 2 & 73 & 2 & 0.975 & 0.905 & 0.975 & 0.905 \\
\hline
\end{tabular}

All samples positive at the STic Expert ${ }^{\circledR}$ HIT test were also tested by Lifecodes PF4 IgG ( $\left.n=114\right)$ or HemosIL ${ }^{\circledR}$ AcuStar HIT-IgG ( $\left.n=96\right)$. Samples were considered positive if both assays were positive at the specified cut-off.

potentially pathogenic HIT antibodies. This has therapeutic relevance, as only these subjects will require an alternative anticoagulation therapy.

In this study we have assessed and compared the validity of three screening assays in confirming or excluding HIT. In agreement with most of the literature on HIT, a low probability 4Ts score is a reliable parameter for excluding HIT [5]. In fact, none of patients with a 4Ts score $<4$ had pathogenic HIT antibodies at HIPA. However, a 4Ts score $\geq 4$ was associated with a PPV of $43 \%$, a value higher than data from a recent meta-analysis, not unexpected as we have observed a prevalence of $25.4 \%$ of confirmed HIT (4Ts score $\geq 4$ and HIPA positive test) [12]. Based on this data, about $75 \%$ of cases with a clinical suspicion did not have HIT. Overall, this data confirms the low reliability of the clinical assessment of patients with an intermediate and high 4Ts score.

We found a higher prevalence of HIT (25\%) compared to previous literature data. A recent meta-analysis [13] evaluating 3068 patients with suspected HIT by both the 4Ts and a functional assay found a pooled estimate of HIT prevalence of only $7 \%-12 \%$. One explanation may be found in the relative underrepresentation (42\%) of cases with a low probability 4Ts score in our casistic compared with previous data where $55.8 \%$ were classified by 4Ts score as having a low probability. Our HIT prevalence is therefore just a confirmation that a low probability pretest scoring system is an accurate tool for ruling out HIT.

As consistently reported in the literature, the IgGspecific ELISA based on polyvinylsulfonate-PF4 antigen at manufacturer recommended cut-off of 0.40 showed the highest sensitivity. Unfortunately, at this cut-off the probability of detecting pathogenic HIT antibodies is rather low as demonstrated by the unacceptable low specificity. Accordingly to previous studies, setting the cut-off at 1 OD improved specificity but the test loses sensitivity [14]. In our experience, the IgG-specific ELISA could be optimized by considering the most northwest point on the correspondent ROC curve, which corresponded to an OD value of 0.92 . Considering only patients with an intermediate/high pretest probability of HIT, this assay increases the probability of HIT from $44 \%$ to $82 \%$. Still, $9.8 \%$ of patients will be misdiagnosed as having HIT.

The IgG-specific LFIA (LFI-STic Expert ${ }^{\circledR}$ HIT) is a rather attractive point-of-care test as it provides results in $<15 \mathrm{~min}$ [15]. In contrast with two recent studies, [8, 15] we found that this assay did not show superior performance compared to the ELISA as it failed to detect 2/29 intermediate-high risk patients with HIT. Basically the test showed the same sensitivity and specificity observed for the IgG-specific ELISA at the optimized OD value of 0.92 . The STic Expert ${ }^{\circledR}$ HIT was negative in two HIPA-positive patients with intermediate and high risk. Although technical issues cannot be excluded, our results do not support an approach based on ruling out HIT in intermediate risk cases with negative STic Expert ${ }^{\circledR}$ HIT, as recently suggested [15]. Therefore, the performance of this assay should be further evaluated in larger clinical studies.

If one relies on a single approach for confirming or excluding HIT, the chemoluminescence assay HemosIL ${ }^{\circledR}$ AcuStar HIT-IgG was associated with the highest performance as it correctly diagnose 90 out of 96 subjects. A cut-off of $1.13 \mathrm{U} / \mathrm{mL}$ further improved the specificity by correctly classifying 92 out of 96 cases, without compromising test sensitivity. The diagnostic accuracy of the test in the set of patients with 4Ts score $\geq 4$ further improved as the probability of HIT increased from $44 \%$ to $89 \%$. The drawback of this otherwise rapid and reliable test relates to the appropriate instrument and the appropriate test cartridges being available in the laboratory. We found the optimization of the threshold did reduce overdiagnosis of HIT although not at the extent reported recently by other authors [16]. Importantly, one HIPA-positive patients who received LMWH prophylaxis after orthopedic surgery did not show anti anti-PF4/H at HemosIL ${ }^{\circledR}$ AcuStar HIT-IgG. Her clinical course was not complicated by thrombotic events. The same patient was negative at STic Expert ${ }^{\circledR}$ 
HIT assay but it would have been correctly diagnosed by ELISA.

Two HIPA-negative patients had a clinical course complicated by thrombotic events. Interestingly, both cases were strongly positive at all three immunoassays. All these subjects had clinical reasons other than HIT to explain thrombocytopenia and thrombosis. In fact one patient has severe sepsis and signs of DIC whereas the other one had idiopathic myelofibrosis and was on hydroxycarbamide therapy. These cases are all examples of the misdiagnosed HIT who may face unnecessary exposure to direct thrombin inhibitors with a risk of major hemorrhage. In these particular patients, discontinuation of heparin and a platelet activation test should be the most reasonable approach. Although our results are concordant with data from the literature that HIPA-negative are likely to have reasons other than HIT to explain thrombocytopenia [17], clinical correlations in this subset of patients are critical as a negative HIPA test does not virtually rule our HIT.

A combinatorial approach has been suggested to yield better performance than use of either assay alone [18]. We found testing patients with the STic Expert ${ }^{\circledR}$ HIT assay and then retesting positive samples with HemosIL ${ }^{\circledR}$ AcuStar HIT-IgG seemed very effective in minimizing HIT overdiagnosis but acceptable sensitivity could not be maintained. Of course, such an approach requires validation in an independent test population.

It is worth noting that we performed inhibition control with high UFH concentrations only in HIPA. This has relevance as we cannot rule out that the inclusion of an inhibition step also in the HemosIL ${ }^{\circledR}$ AcuStar HIT-IgG and IgG-specific ELISA (not feasible for the STic Expert ${ }^{\circledR}$ HITassay), may have reduced the rate of false-positive results.

It is critical to stress that none of the assays evaluated in this study could achieve dual 100\% sensitivity and specificity, meaning that some patients with HIT will be missed and others without HIT will be misdiagnosed as positive. To minimize this issue, clinical guidance by 4Ts is therefore highly recommended.

In conclusion, although none of the immunological assays can be considered a substitute for the functional assay, a single test approach based on the HemosIL ${ }^{\circledR}$ AcuStar HIT with optimized thresholds represents the best strategy in the attempt to reduce overdiagnosis of HIT without compromising test sensitivity.

Author contributions: All the authors have accepted responsibility for the entire content of this submitted manuscript and approved submission.

Financial support: None declared.

Employment or leadership: None declared.
Honorarium: None declared.

Competing interests: The funding organization(s) played no role in the study design; in the collection, analysis, and interpretation of data; in the writing of the report; or in the decision to submit the report for publication.

\section{References}

1. Amiral J, Bridey F, Dreyfus M, Vissoc AM, Fressinaud E, Wolf M, et al. Platelet factor 4 complexed to heparin is the target for antibodies generated in heparin-induced thrombocytopenia. Thromb Haemost 1992;68:95-6.

2. Rauova L, Poncz M, McKenzie SE, Reilly MP, Arepally G, Weisel JW, et al. Ultralarge complexes of PF4 and heparin are central to the pathogenesis of heparin-induced thrombocytopenia. Blood 2005;105:131-8.

3. Warkentin TE, Greinacher A, Gruel Y, Aster RH, Chong BH. Laboratory testing for heparin-induced thrombocytopenia: a conceptual framework and implications for diagnosis. J Thromb Haemost 2011;9:2498-500.

4. Warkentin TE, Sheppard JI, Moore JC, Sigouin CS, Kelton JG. Quantitative interpretation of optical density measurements using PF4-dependent enzyme-immunoassays. J Thromb Haemost 2008;6:1304-12.

5. Lo GK, Juhl D, Warkentin TE, Sigouin CS, Eichler P, Greinacher A. Evaluation of pretest clinical score (4 T's) for the diagnosis of heparin-induced thrombocytopenia in two clinical settings. J Thromb Haemost 2006;4:759-65.

6. Greinacher A, Michels I, Kiefel V, Mueller-Eckhardt C. A rapid and sensitive test for diagnosing heparin-associated thrombocytopenia. Thromb Haemost 1991;66:734-6.

7. Legnani C, Cini M, Pili C, Boggian O, Frascaro M, Palareti G. Evaluation of a new automated panel of assays for the detection of anti-PF4/heparin antibodies in patients suspected of having heparin-induced thrombocytopenia. Thromb Haemost 2010;104:402-9.

8. Sachs UJ, von Hesberg J, Santoso S, Bein G, Bakchoul T. Evaluation of a new nanoparticle-based lateral-flow immunoassay for the exclusion of heparin-induced thrombocytopenia (HIT). Thromb Haemost 2011;106:1197-202.

9. DeLong ER, DeLong DM, Clarke-Pearson DL. Comparing the areas under two or more correlated receiver operating characteristic curves: a nonparametric approach. Biometrics 1988;44:837-45.

10. Warkentin TE, Sheppard JA, Horsewood P, Simpson PJ, Moore JC, Kelton JG. Impact of the patient population on the risk for heparin-induced thrombocytopenia. Blood 2000;96:1703-8.

11. Pouplard C, May MA, lochmann S, Amiral J, Vissac AM, Marchand M, et al. Antibodies to platelet factor 4-heparin after cardiopulmonary bypass in patients anticoagulated with unfractionated heparin or a low-molecular-weight heparin: clinical implications for heparin-induced thrombocytopenia. Circulation 1999;99:2530-6.

12. Cuker A, Gimotty PA, Crowther MA, Warkentin TE. Predictive value of the 4Ts scoring system for heparin-induced thrombocytopenia: a systematic review and meta-analysis. Blood 2012;120:4160-7. 
13. Zwicker Jl, Uhl L, Huang WY, Shaz BH, Bauer KA. Thrombosis and ELISA optical density values in hospitalized patients with heparininduced thrombocytopenia. J Thromb Haemost 2004;2:2133-7.

14. Kolde HJ, Habrecht U, von Hesberg J, Panzer S, Seidel H, Bakchoul T, et al. Multicentric validation of a rapid assay for heparin-induced thrombocytopenia with different specimen types. Blood Coagul Fibrinolysis 2014;25:6-9.

15. Leroux D, Hezard N, Lebreton A, Bauters A, Suchon P, de Maistre $E$, et al. Prospective evaluation of a rapid nanoparticle-based lateral flow immunoassay (STic Expert HIT) for the diagnosis of heparin-induced thrombocytopenia. $\mathrm{Br} J$ Haematol 2014;166:774-82.
16. Minet V, Baudar J, Bailly N, Douxfils J, Laloy J, Lessire S, et al. Rapid exclusion of the diagnosis of immune HIT by AcuStar HIT and heparin-induced multiple electrode aggregometry. Thromb Res 2014;133:1074-8.

17. Greinacher A, Juhl D, Strobel U, Wessel A, Lubenow N, Selleng K et al. Heparin-induced thrombocytopenia: a prospective study on the incidence, platelet-activating capacity and clinical significance of antiplatelet factor 4/heparin antibodies of the IgG, IgM, and IgA classes. J Thromb Haemost 2007;5:1666-73.

18. Cuker A, Rux AH, Hinds JL, Dela Cruz M, Yarovoi SV, Brown IA, et al. Novel diagnostic assays for heparin-induced thrombocytopenia. Blood 2013;121:3727-32. 